\title{
Reionization on Large Scales. I. A Parametric Model Constructed From Radiation-hydrodynamic Simulations
}

\section{Citation}

Battaglia, N., H. Trac, R. Cen, and A. Loeb. 2013. "REIONIZATION ON LARGE SCALES. I. A PARAMETRIC MODEL CONSTRUCTED FROM RADIATION-HYDRODYNAMIC SIMULATIONS." The Astrophysical Journal 776 (2): 81. https://doi.org/10.1088/0004-637x/776/2/81.

\section{Permanent link}

http://nrs.harvard.edu/urn-3:HUL.InstRepos:41393181

\section{Terms of Use}

This article was downloaded from Harvard University's DASH repository, and is made available under the terms and conditions applicable to Other Posted Material, as set forth at http:// nrs.harvard.edu/urn-3:HUL.InstRepos:dash.current.terms-of-use\#LAA

\section{Share Your Story}

The Harvard community has made this article openly available. Please share how this access benefits you. Submit a story.

Accessibility 


\title{
REIONIZATION ON LARGE SCALES. I. A PARAMETRIC MODEL CONSTRUCTED FROM RADIATION-HYDRODYNAMIC SIMULATIONS
}

\author{
N. Battaglia ${ }^{1}$, H. Trac ${ }^{1}$, R. Cen $^{2}$, And A. Loeb ${ }^{3}$ \\ ${ }^{1}$ McWilliams Center for Cosmology, Wean Hall, Carnegie Mellon University, 5000 Forbes Avenue, Pittsburgh PA 15213, USA \\ ${ }^{2}$ Department of Astrophysical Sciences, Princeton University, Princeton, NJ 08544, USA \\ ${ }^{3}$ Harvard-Smithsonian Center for Astrophysics, Cambridge, MA 02138, USA \\ Received 2012 November 13; accepted 2013 August 9; published 2013 October 1
}

\begin{abstract}
We present a new method for modeling inhomogeneous cosmic reionization on large scales. Utilizing highresolution radiation-hydrodynamic simulations with $2048^{3}$ dark matter particles, $2048^{3}$ gas cells, and 17 billion adaptive rays in a $L=100 \mathrm{Mpc} h^{-1}$ box, we show that the density and reionization redshift fields are highly correlated on large scales $\left(\gtrsim 1 \mathrm{Mpc} h^{-1}\right)$. This correlation can be statistically represented by a scale-dependent linear bias. We construct a parametric function for the bias, which is then used to filter any large-scale density field to derive the corresponding spatially varying reionization redshift field. The parametric model has three free parameters that can be reduced to one free parameter when we fit the two bias parameters to simulation results. We can differentiate degenerate combinations of the bias parameters by combining results for the global ionization histories and correlation length between ionized regions. Unlike previous semi-analytic models, the evolution of the reionization redshift field in our model is directly compared cell by cell against simulations and performs well in all tests. Our model maps the high-resolution, intermediate-volume radiation-hydrodynamic simulations onto lower-resolution, larger-volume $N$-body simulations $\left(\gtrsim 2 \mathrm{Gpc}^{-1}\right)$ in order to make mock observations and theoretical predictions.
\end{abstract}

Key words: cosmology: theory - intergalactic medium - large-scale structure of universe - methods: numerical

Online-only material: color figures

\section{INTRODUCTION}

When the first stars and galaxies started emitting photons, they began ionizing the surrounding cold and neutral hydrogen of the intergalactic medium (IGM). This started the phase transition of the universe known as the Epoch of Reionization (EoR; Loeb \& Furlanetto 2013). The process of reionization is inhomogeneous and produces at least two major observable sources: the neutral hydrogen atoms and the ionized electrons. Precise theoretical models of the EoR on Gpc scales are necessary for interpreting the information from the observable imprints left by these sources in order to gain an understanding of the first stars and the initial stages of galaxy formation (e.g., Furlanetto et al. 2006; Morales \& Wyithe 2010 and references therein).

Neutral hydrogen atoms are observed in both absorption and emission. Current constraints from absorption measurements come from observations of zero transmission of the rest-frame Ly $\alpha$ flux at $z \gtrsim 6$ in spectra of high-redshift quasars, which suggest that the EoR is completed by $z \sim 6$ (Fan et al. 2006). However, it is possible for these constraints to be consistent with reionization completing at a higher redshift (e.g., Oh \& Furlanetto 2005; Lidz et al. 2006). The neutral hydrogen emission is observable through the redshifted $21 \mathrm{~cm}$ signal that originates from its hyperfine transition (e.g., Scott \& Rees 1990; Shaver et al. 1999; Zaldarriaga et al. 2004). There are several experiments currently searching for the $21 \mathrm{~cm}$ signal at $z>6$, such as the Murchison Wide Field Array (MWA ${ }^{4}$; Bowman et al. 2005), the Giant Meterwave Telescope $\left(\right.$ GMRT $^{5}$; Pen et al. 2009), the Low Frequency Array (LOFAR ${ }^{6}$; Harker et al. 2010),

\footnotetext{
4 http://www.mwatelescope.org

5 http://gmrt.ncra.tifr.res.in

6 http://www.lofar.org
}

and the Precision Array for Probing the Epoch of Reionization (PAPER ${ }^{7}$; Parsons et al. 2010). Using the $21 \mathrm{~cm}$ signal, the experiment EDGES $^{8}$ reported a lower limit to the duration of reionization, $\Delta_{\mathrm{z}}>0.06$ (Bowman \& Rogers 2012). Future $21 \mathrm{~cm}$ experiments, like the proposed Square Kilometer Array (SKA ${ }^{9}$; Mellema et al. 2013), have the potential to measure this signal across several frequencies and provide tomographic information on the EoR.

The free electrons are observed through their scattering of cosmic microwave background (CMB) photons. This scattering can be seen on large scales in polarization CMB or on small scales in the CMB secondary anisotropies, such as kinetic Sunyaev-Zel'dovich (kSZ) signal from the EoR (Gruzinov \& Hu 1998; Knox et al. 1998; Valageas et al. 2001; Santos et al. 2003; Zahn et al. 2005; McQuinn et al. 2005; Iliev et al. 2007; Mesinger et al. 2011). There are constraints on the optical depth to the EoR (e.g., Larson et al. 2011) from polarization measurements of the CMB. Assuming a step function or a hyperbolic tangent function for the ionization history, the seven-year Wilkinson Microwave Anisotropy Probe $(W M A P)$ data implies that the reionization redshift is $10.5 \pm 1.2$ (68\% CL). Constraints have also come from multi-frequency high resolution $\mathrm{CMB}$ experiments that measure the power spectrum of CMB secondary anisotropies to great precision, where contributions to $\mathrm{kSZ}$ power from EoR are the largest. The South Pole Telescope (SPT ${ }^{10}$; Zahn et al. 2012) placed a model dependent upper limit on the duration of reionization from their multifrequency measurements of the high $\ell$ power spectrum and

\footnotetext{
7 http://eor.berkeley.edu

8 http://www.haystack.mit.edu/ast/arrays/Edges

9 http://www.skatelescope.org

$10 \mathrm{http}: / /$ pole.uchicago.edu
} 
future results from the Atacama Cosmology Telescope $\left(\mathrm{ACT}^{11}\right)$ are expected to place similar constraints. The next generation high-resolution CMB experiments ACT with polarization (ACTpol) and South Pole Telescope with polarization (SPT-pol) will precisely measure the secondary anisotropies of the CMB in both temperature and polarization, which will provide tighter constraints on the EoR.

For the EoR experiments listed above and future ones, the amount of understanding gained on these first ionizing sources and the initial stages of galaxy evolution will depend upon the accuracy of the theoretical models for the EoR. The main challenge in the EoR theory is providing an accurate model of the IGM, the sources, and the sinks of ionizing photons while having a large enough volume $>1\left(\mathrm{Gpc} h^{-1}\right)^{3}$ to statistically sample the $\mathrm{H}$ I regions and construct mock observations on the angular scales required by the current and future EoR experiments.

There are two standard approaches when modeling the EoR: radiative transfer simulations with various implementations for hydrodynamics and gas physics (e.g., Gnedin \& Abel 2001; Ciardi et al. 2001; Maselli et al. 2003; Alvarez et al. 2006; Mellema et al. 2006; Iliev et al. 2006; Trac \& Cen 2007; McQuinn et al. 2007; Trac et al. 2008; Aubert \& Teyssier 2008; Altay et al. 2008; Croft \& Altay 2008; Finlator et al. 2009; Petkova \& Springel 2009) and semi-analytic models (e.g., Furlanetto et al. 2004; Zahn et al. 2005, 2007; Mesinger \& Furlanetto 2007; Geil \& Wyithe 2008; Alvarez et al. 2009; Thomas et al. 2009; Choudhury et al. 2009; Santos et al. 2010; Mesinger et al. 2011). In these semi-analytic models a region is fully ionized if the simple relation, $\zeta F_{\text {coll }} \geqslant 1$ is satisfied. Here $\zeta$ is an efficiency parameter and $F_{\text {coll }}$ is the collapse fraction, which is calculated via the excursion set formalism (Bond et al. 1991), or applied to a three-dimensional (3D) realization of a density field (e.g., Zahn et al. 2005). Semi-analytic models capture the generic properties of the EoR, but in order to capture the complex non-linear nature of the EoR radiative transfer simulations are required.

The advantage of the current fully hydrodynamic, highresolution simulations with radiative transfer (implemented either in post processing or during the simulation) is that they probe the relevant scales to resolve sources of ionizing photons and their sinks then trace these photons through an inhomogeneous IGM (Trac \& Gnedin 2011). However, running full hydrodynamic simulations with radiative transfer on large enough scales to capture a representative sample of ionizing sources and with enough small-scale resolution to also capture all the physics of reionization are currently not possible due to the overwhelming computational demands of such calculations. Thus, all of the simulations to date have been restricted to smaller box-sizes. Recent work by Zahn et al. (2011) ran several convergence tests between these two types of approaches that model the EoR. For all the models in their study, they found that the results from the models are within tens of percent of each other. However, in these comparisons the parameters of semianalytic models were adjusted to match the ionization fractions of the simulations at the redshifts of interest.

In this paper, we present a substantially more accurate semianalytical model that is statistically informed by simulations with radiative transfer and hydrodynamics. The implementation of this model is fast, versatile, and easily applied to large $N$-body simulations, thus it can be scaled up to the large volumes required by the current and future EoR experiments

11 http://www.princeton.edu/act without loss of accuracy. This paper is the first in a series that explores observables from the EoR produced via our model. We focus on CMB-related observables in Natarajan et al. (2013); Battaglia et al. (2013); and the $21 \mathrm{~cm}$ in P. La Plante et al. (2013, in preparation). In Section 2, we present our fast semianalytical model and the simulations it is calibrated on. Section 3 compares the model to the simulations on a cell by cell basis. We show results on the global reionization history and the typical correlation between ionized regions in Section 4. We compare to previous work and discuss caveats to our model in Section 5 and conclude in Section 6. Throughout the paper, we adopt the concordance cosmological parameters: $\Omega_{\mathrm{m}}=0.27, \Omega_{\Lambda}=0.73$, $\Omega_{\mathrm{b}}=0.045, h=0.7, n_{\mathrm{s}}=0.96$, and $\sigma_{8}=0.80$.

\section{METHODOLOGY}

We present a novel semi-analytic model for calculating the evolution of the 3D ionization field in large volumes $\gtrsim\left(\mathrm{Gpc} h^{-1}\right)^{3}$, which is currently not attainable with direct simulations. Using radiation-hydrodynamic simulations, we demonstrate that the redshift at which a volume element is ionized can be calculated by filtering a nonlinear density field with a simple parametric function. Our method can be used to map high-resolution, intermediate-volume radiationhydrodynamic simulations onto lower-resolution, larger-volume $\mathrm{N}$-body simulations in order to make mock observations and theoretical predictions. In addition, the model parameters can be varied away from the fiducial values in order to explore the reionization parameter space (e.g., the timing and duration of the EoR).

\subsection{Hydrodynamic Simulations with Radiative Transfer}

We adopt the hybrid approach in simulating cosmic reionization previously described in Trac et al. (2008). First, a high-resolution $N$-body simulation is used to evolve the matter distribution and track the formation of dark matter halos. The resulting halo catalogs are used to develop a subgrid model for high-redshift radiation sources. Second, direct RadHydro (radiative transfer + hydrodynamic $+N$-body) simulations are used to simultaneously solve the coupled evolution of the dark matter, baryons, and radiation.

A particle-particle-particle-mesh $\left(\mathrm{P}^{3} \mathrm{M}\right)$ code is used to run a high-resolution simulation with $3072^{3}$ dark matter particles in a $100 \mathrm{Mpc} h^{-1}$ comoving box. A spherical overdensity halo finder is used on the fly to identify collapsed dark matter halos with average densities $\bar{\rho}(z)$ equal to 200 times the average cosmic density $\rho_{m}(z)$. With a particle mass resolution of $2.58 \times$ $10^{6} M_{\odot} h^{-1}$, we can reliably locate dark matter halos down to the atomic cooling limit $\left(T \sim 10^{4} \mathrm{~K}, M \sim 10^{8} M_{\odot} h^{-1}\right)$. The halos are then populated with radiation sources using a halo model similar to that previously described in Trac \& Cen (2007).

For each atomic cooling halo, the star formation rate is calculated as

$$
\frac{d M_{*}}{d t}=c_{*} \frac{M}{t_{\mathrm{dyn}}},
$$

where $c_{*}$ is an average star formation efficiency and the redshiftdependent halo dynamical time is defined as

$$
t_{\text {dyn }}(z)=\sqrt{\frac{3}{32 G \bar{\rho}(z)}} .
$$



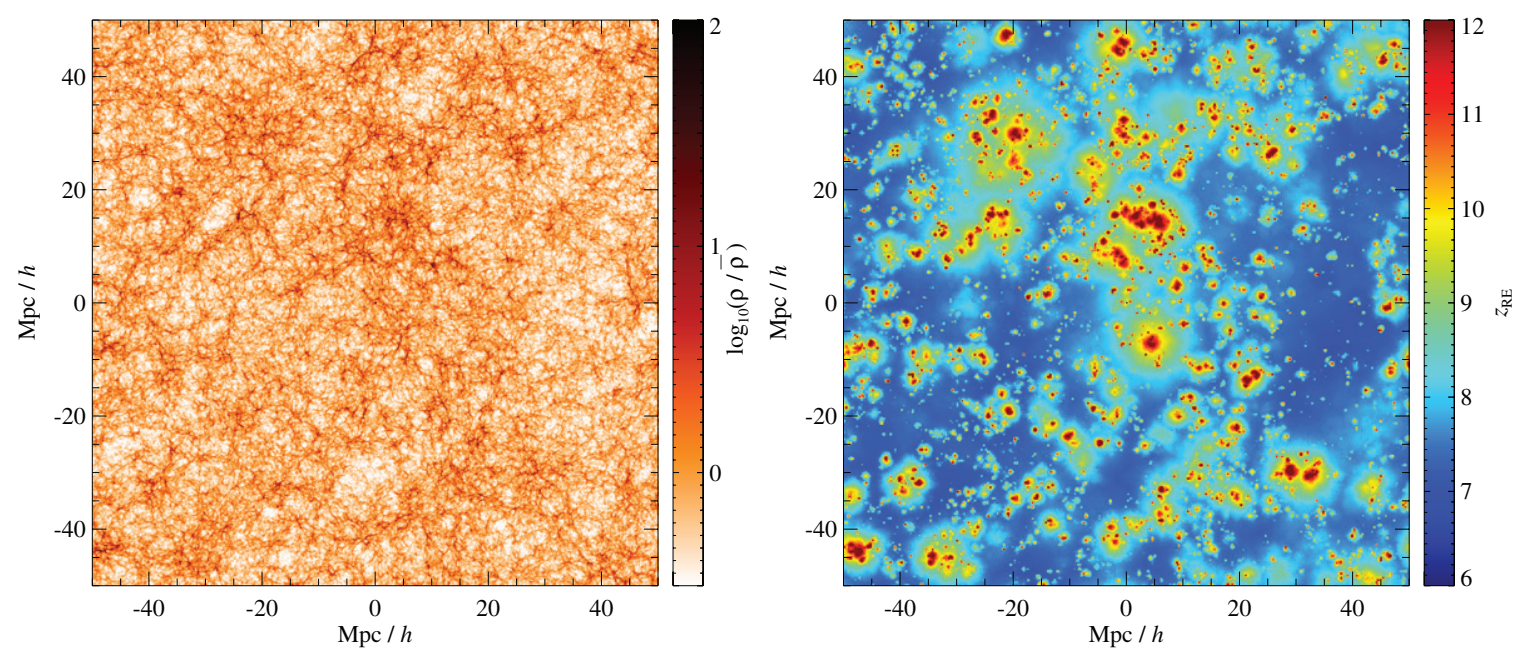

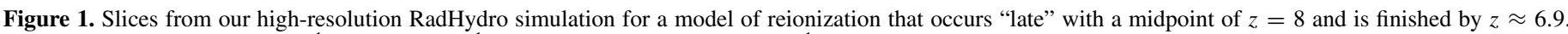

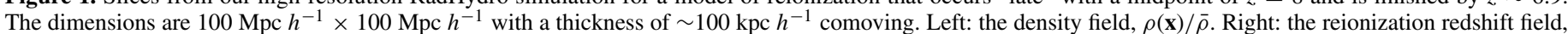
$z_{\mathrm{RE}}(\mathbf{x})$. Large-scale, overdense regions near sources are generally ionized earlier than large-scale, underdense regions far from sources.

(A color version of this figure is available in the online journal.)

The specific ionizing photon rate is then calculated as

$$
\frac{d N}{d t d v}=f_{\mathrm{esc}} N_{m v} \frac{d M_{*}}{d t},
$$

where $f_{\text {esc }}$ is an average radiation escape fraction and $N_{m v}(v)$ is the number of photons produced per unit mass of star formation per unit frequency. The overall abundance of ionizing photons depends on the product of $c_{*}$ and $f_{\text {esc }}$, both of which are still poorly constrained by current observations and simulations. In practice, this quantity is varied to adjust the abundance of ionizing photons and thereby change the timing and duration of the reionization process.

The RadHydro code combines a cosmological hydrodynamic code (moving frame hydrodynamics + particle-mesh $N$-body; Trac \& Pen 2004) with an adaptive ray-tracing radiative transfer algorithm (Trac \& Cen 2007). The ray-tracing algorithm has adaptive splitting and merging and utilizes a two-level radiative transfer grid scheme to obtain better resolution and scaling. We have run two moderate-resolution RadHydro simulations each with $2048^{3}$ dark matter particles, $2048^{3}$ gas cells, and 17 billion adaptive rays. Five frequencies above the hydrogen ionizing threshold of $13.6 \mathrm{eV}$ are tracked for each ray. The photoionization and photoheating rates for each cell are calculated from the incident radiation flux and used in the nonequilibrium solvers for the ionization and energy equations. The RadHydro and $N$-body simulations were run using the Blacklight supercomputer at the Pittsburgh Supercomputing Center (PSC).

In the first RadHydro simulation, reionization occurs earlier with a midpoint of $z \approx 10$ (mass and volume-weighted ionization fractions are $\approx 0.5$ ) and is effectively completed by $z \approx 8.7$ (radiation filling factor of the radiation-hydrodynamic grid reaches unity). This early reionization model has a Thomson optical depth for electron scattering $\tau \approx 0.088$, which is in good agreement with current observational constraints. From the WMAP seven-year results, the Thomson optical depth is $\tau=0.088 \pm 0.015$ assuming instantaneous reionization and $\tau=0.087 \pm 0.015$ if the width of reionization is allowed to vary (Larson et al. 2011). In the second simulation, reionization occurs later with a midpoint of $z=8$ and is finished by $z \approx 6.9$. The late model has $\tau_{\mathrm{e}} \approx 0.067$, which is lower but within $2 \sigma$ of the WMAP best-fit value.
In the two basic simulations, the radiative transfer of the ionizing photons proceeded such that large-scale, overdense regions near sources are generally ionized earlier than large-scale, underdense regions far from sources. The subgrid model for sources only included high-redshift galaxies with Population II stars (Schaerer 2003) since they are expected to provide the dominant contribution to the ionizing photon budget. The simulations do not include reionization by Population III stars or X-ray sources, which primarily affect the earliest phase of the EoR (e.g., Furlanetto et al. 2006). We also neglect additional clumping and self-shielding of small-scale dense absorbers such as mini halos (e.g., Shapiro et al. 2004) or Lyman limit systems (e.g., Gnedin \& Fan 2006). We will explore other reionization scenarios using different models for sources and sinks in future work.

\subsection{Semi-analytic Model}

For every cell in the RadHydro simulations, we record the redshift at which it ionizes and construct a reionization redshift field, $z_{\mathrm{RE}}(\mathbf{x})$. Figure 1 shows a slice of the density and the reionization redshift fields for the late reionization scenario. For the purposes of this work, regions that are greater than $90 \%$ ionized are considered to be finished reionizing, but we obtain nearly identical statistical quantities when we chose $50 \%$ (cf. Figure 2). Defining a threshold for when cells are considered to be ionized is arbitrary, since there is a sharp transition between the onset of ionization and completion within a cell. Most cells approach $100 \%$ ionization but never reach it due to recombinations. Our results are consistent with those of Trac et al. (2008), who used a 99\% ionization threshold.

Figure 1 shows the overdensity field at $z=8$ and the corresponding reionization field from the simulations, which clearly illustrates that the reionization redshift is associated with the density. The two fields are highly correlated since large-scale overdensities near sources are generally ionized earlier than large-scale, under dense regions far from sources (Barkana \& Loeb 2004). Other semi-analytical approaches implicitly invoke this association when constructing their models (e.g., Furlanetto et al. 2004; Zahn et al. 2005), and Figure 1 illustrates that this assumption is fairly accurate down to Mpc scales. Our method quantifies the correlation between the reionization redshift and 

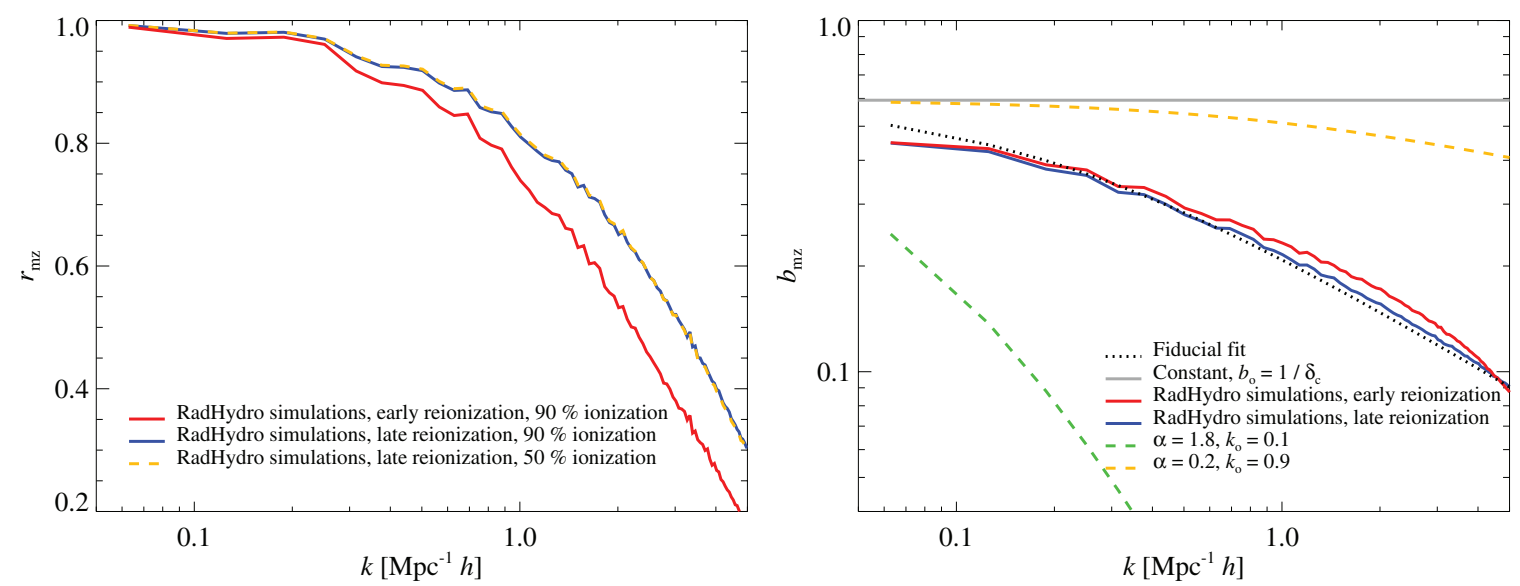

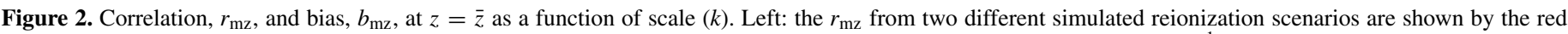

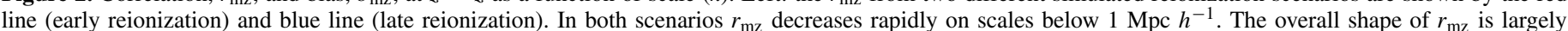

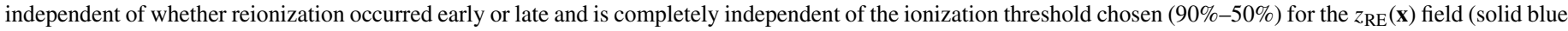

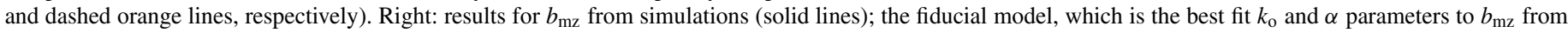

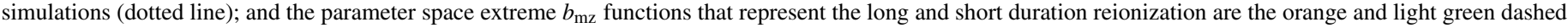

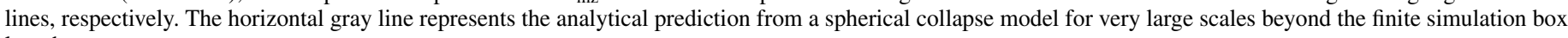
length.

(A color version of this figure is available in the online journal.)

density in our RadHydro simulations and uses this correlation and its bias to construct reionization fields from any density field. First, we define the following fluctuation fields

$$
\delta_{\mathrm{m}}(\mathbf{x}) \equiv \frac{\rho(\mathbf{x})-\bar{\rho}}{\bar{\rho}},
$$

and

$$
\delta_{\mathrm{z}}(\mathbf{x}) \equiv \frac{\left[1+z_{\mathrm{RE}}(\mathbf{x})\right]-[1+\bar{z}]}{1+\bar{z}},
$$

where $\bar{\rho}$ is the mean matter density and $\bar{z}$ is the mean value for the $z_{\mathrm{RE}}(\mathbf{x})$ field. This construction has the advantage that we removed mean redshift dependences of $z_{\mathrm{RE}}(\mathbf{x})$ and these fields are dimensionless. Then we quantify their correlation using two point statistics $\left\langle\delta_{i} \delta_{j}\right\rangle=P_{i j}(k)$ in Fourier space (i.e., power spectra and cross spectra) assuming isotropy and we calculate their cross correlation

$$
r_{\mathrm{mz}}(k)=\frac{P_{\mathrm{mz}}(k)}{\sqrt{P_{\mathrm{zz}}(k) P_{\mathrm{mm}}(k)}},
$$

and linear bias,

$$
b_{\mathrm{mz}}(k)=\sqrt{\frac{P_{\mathrm{zz}}(k)}{P_{\mathrm{mm}}(k)}},
$$

at $z=\bar{z}$. Figure 2 shows that these fields are highly correlated on scales above $1 \mathrm{Mpc} h^{-1}$ and this correlation decreases as one moves to smaller scales. Similar cross correlations between ionization fields and density are calculated in Zahn et al. (2011) for fixed redshifts. As a result of $r_{\mathrm{mz}}(k)$ being highly correlated on scales above $1 \mathrm{Mpc} h^{-1}$, just knowing the bias between these two fields allows one to construct either field from the other by filtering the initial field with the bias. The newly constructed field will statistically match the results from the simulations. We chose to calculate a linear bias (cf. Equation (7)), since this bias matches well the $z_{\mathrm{RE}}(\mathbf{x})$ from the simulations on scales $>1 \mathrm{Mpc} h^{-1}$ (cf. Figure 3). Going to higher-order correlations and bias models should improve this comparison, but it is not necessary to capture the global features at the accuracy we are interested in; such extensions are left for future work. We find that the linear bias can be fit by a three-parameter function (cf. Figure 2). The simple functional form for the bias factor is

$$
b_{\mathrm{mz}}(k)=\frac{b_{\mathrm{o}}}{\left(1+k / k_{\mathrm{o}}\right)^{\alpha}},
$$

with the three free parameters being $b_{\mathrm{o}}, k_{\mathrm{o}}$, and $\alpha$. A simple description for the parameters is as follows: $b_{\mathrm{o}}$ is the bias amplitude, $k_{\mathrm{o}}$ is the scale threshold, and $\alpha$ is the asymptotic exponent. We fit $k_{\mathrm{o}}$ and $\alpha$ to results from the simulations using least-squares fitting with the correlation function $\left(r_{\mathrm{mz}}\right)$ as weight (cf. Figure 2). This weighting emphasizes the scales where $\delta_{\mathrm{z}}$ and $\delta_{\mathrm{m}}$ are highly correlated and down weights the small scales. The best-fit values for the $b_{\mathrm{mz}}$ are $k_{\mathrm{o}}=0.185 \mathrm{Mpc} h^{-1}$ and $\alpha=0.564$. Hereafter, we refer to these parameter values as the fiducial bias parameters. We show in Figure 2 that our parametric function with these fiducial parameters is comparable to the calculated simulation bias. Also, we explore the parameter space of $k_{\mathrm{o}}$ and $\alpha$ and allow them to vary about the fiducial parameters.

The third parameter, $b_{0}$, which is the bias amplitude on the largest scales, is not fit for, since the simulations that we ran have finite box sizes and these scales extend beyond their box size. Figure 2 shows that $b_{\mathrm{mz}}$ is asymptotically approaching some value for $b_{\mathrm{o}}$, but fitting for this value would be inaccurate and degenerate with $k_{\mathrm{o}}$ and $\alpha$. Instead we refer to previous analytic work by Barkana \& Loeb (2004) where this large-scale bias is derived using the excursion set formalism (i.e., extended Press-Schechter formalism; Bond et al. 1991). They show that on these large scales, the differences in the redshift of collapse and reionization for various over densities are related via

$$
\delta_{\mathrm{z}}(k \rightarrow 0)=\frac{\delta_{\mathrm{m}}(k \rightarrow 0)}{\delta_{\mathrm{c}}} .
$$

Here $\delta_{\mathrm{c}}=1.68$ is the critical over-density threshold. Throughout this work we use the value $b_{\mathrm{o}}=1 / \delta_{\mathrm{c}}=0.593$.

\subsection{Implementation}

We used a $\mathrm{P}^{3} \mathrm{M} N$-body code to evolve $2048^{3}$ dark matter particles in a $2 \mathrm{Gpc} h^{-1}$ box to generate the over density 

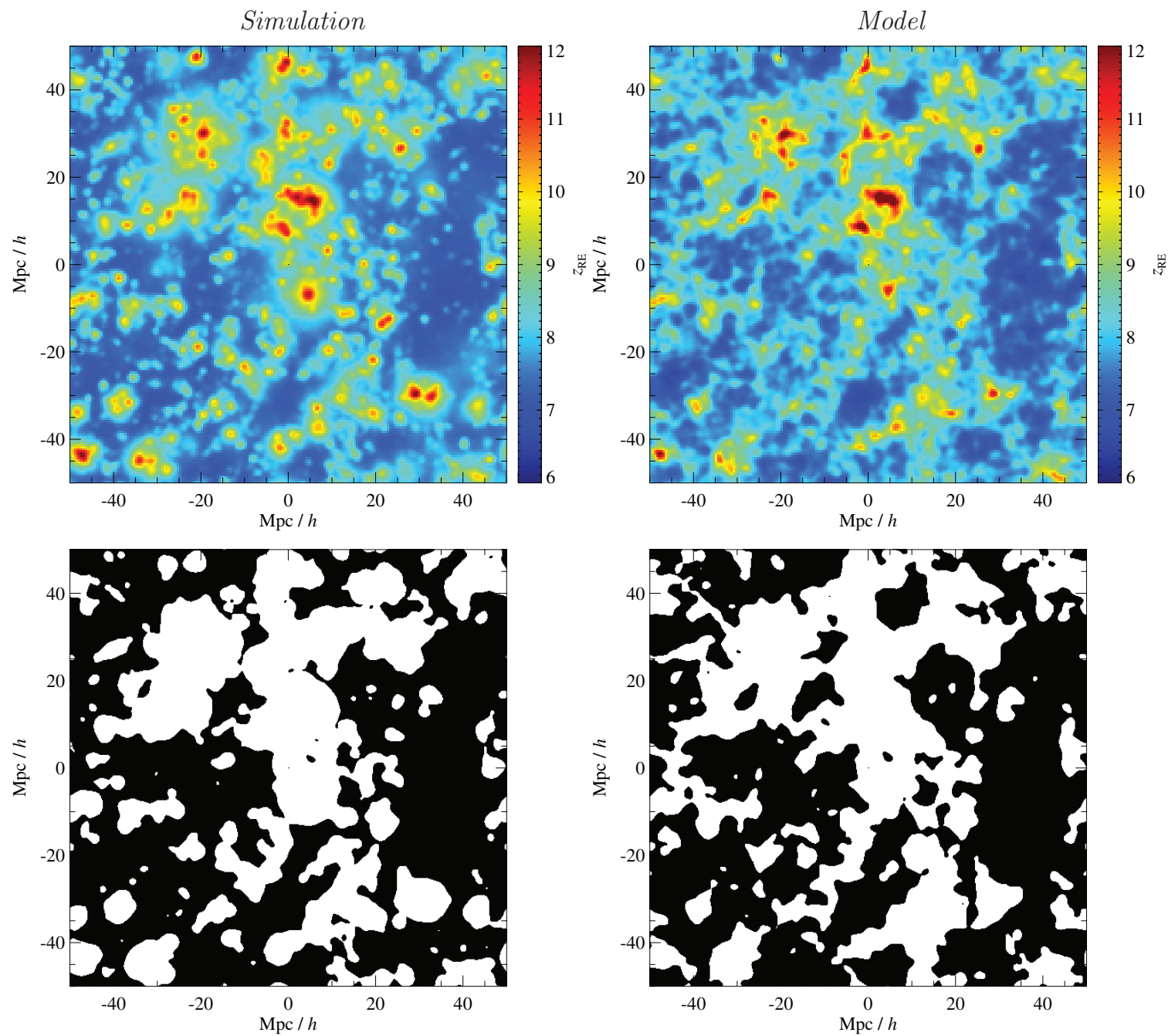

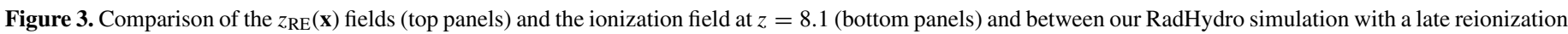

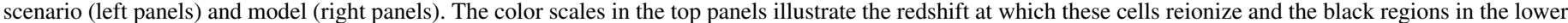

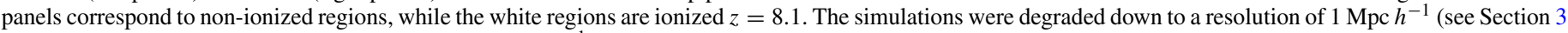

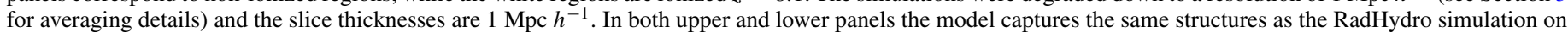
large scales. The $z_{\mathrm{RE}}(\mathbf{x})$ fields differ on small scales in the void and filament regions, where the our method tends to predict earlier redshifts of reionization.

(A color version of this figure is available in the online journal.)

fields, $\delta_{\mathrm{m}}$, down to $z=5.5$, but in principal any density field can be used; for example, one from second-order Lagrangian perturbation theory (e.g., Scoccimarro \& Sheth 2002). We construct the $z_{\mathrm{RE}}(\mathbf{x})$ field with the following steps.

1. We Fourier transform $\delta_{\mathrm{m}}$ at $z=\bar{z}$ and obtain $\tilde{\delta}_{\mathrm{m}}(k)$.

2. We multiply $\tilde{\delta}_{\mathrm{m}}(k)$ with a filter consisting of three elements: (1) a cubical top-hat filter, $\Xi(k)$, which deconvolves the smoothing used to construct $\delta_{\mathrm{m}}$ from simulations; (2) a Fourier transform of a real space top-hat filter $\Theta(k)$, which smoothes $\delta_{\mathrm{m}}$ to resolution of $1 \mathrm{Mpc} h^{-1}$; and (3) the bias function from Equation (8). The assembled filter takes this form:

$$
W_{\mathrm{z}}(k) \equiv \frac{b_{\mathrm{mz}}(k) \Theta(k)}{\Xi(k)},
$$

and this multiplication converts $\tilde{\delta}_{\mathrm{m}}(k)$ to $\tilde{\delta}_{\mathrm{z}}(k)$.

3. We inverse-Fourier transform $\tilde{\delta}_{\mathrm{z}}(k)$ back to real space to obtain the $\delta_{\mathrm{z}}$ field.

4. We convert the $\delta_{\mathrm{z}}$ field to the $z_{\mathrm{RE}}(\mathbf{x})$ field using Equation (5) with the same $\bar{z}$ as the density field; here $\bar{z}$ essentially sets the midpoint of reionization.
Following these steps results in a complete ionization history for the density field used and from the $z_{\mathrm{RE}}(\mathbf{x})$ field. Therefore, one can construct 3D realizations of the ionization or neutral hydrogen fields at a given redshift. The entire process is quick, since it only requires Fourier transforms, which can be done with any fast Fourier transform software and will scale like $N \log (N)$, where $N$ is the number of cells. We found that $\mathrm{I} / \mathrm{O}$ of these simulation data files dominates the total time when producing a $z_{\mathrm{RE}}(\mathbf{x})$ field. This quick model allows one to easily explore the parameter space of $k_{\mathrm{o}}, \alpha$, and $\bar{z}$.

\section{COMPARISON TO SIMULATIONS}

We compare the results from the RadHydro simulations for the reionization redshift fields, $z_{\mathrm{RE}}(\mathbf{x})$, to our model predictions that were constructed field from the same simulated density fields. Similar to Zahn et al. (2011), we also compare their ionization fields and morphology statistics at fixed redshifts. Unlike the previous comparisons between simulations and semianalytic models, some of our comparisons of the $z_{\mathrm{RE}}(\mathbf{x})$ fields are computed at the cell by cell level. Furthermore, the $b_{\mathrm{mz}}$ 


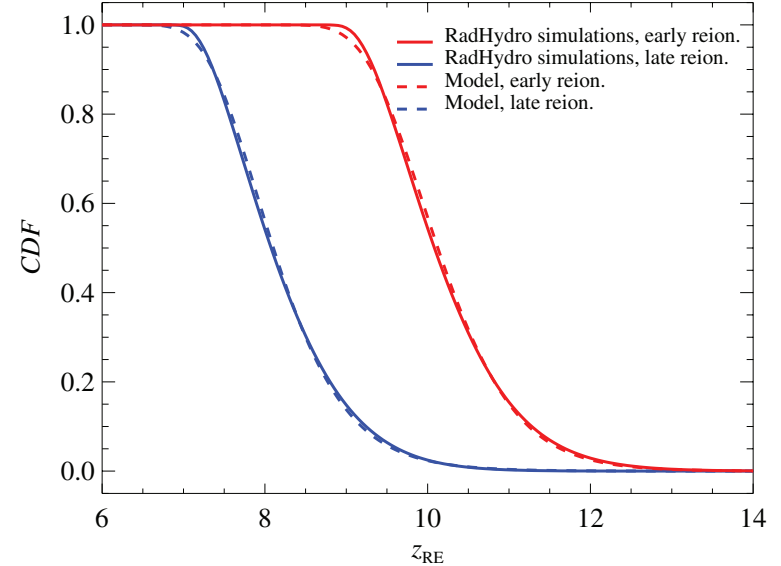

Figure 4. Comparison of the cumulative distribution function (CDF) for the $z_{\mathrm{RE}}(\mathbf{x})$ field between RadHydro simulations (early and late reionization scenarios, represented by the red and blue lines, respectively) and our model predictions constructed from the same density fields as the simulations. Our models deviate from the RadHydro simulations at the beginning and end of reionization, since the models are constructed for density fields at $\bar{z}$. Overall this accounts for percent level changes in the duration of reionization; the exact amount will depend on the definition for the duration.

(A color version of this figure is available in the online journal.)

parameters used to in this comparison are not tuned to match a particular ionization fraction at a given redshift. They are calculated from the RadHydro simulations. Our comparisons go beyond bubble size and distribution tests, since we look at the redshift evolution of these quantities as well. In order to make these direct comparisons, the simulations must be smoothed to the scales on which we apply our model $\gtrsim 1 \mathrm{Mpc} h^{-1}$, since they are at a higher resolution than $1 \widetilde{\mathrm{Mpc}} h^{-1}$. The simulations are smoothed by convolving them with a cubical top-hat filter, which degrades the resolution of their density and $z_{\mathrm{RE}}(\mathbf{x})$ fields to $\sim 1 \mathrm{Mpc} h^{-1}$. After smoothing the simulations, we find that the same structures in the $z_{\mathrm{RE}}(\mathbf{x})$ field slice seen in Figure 1 are still visible in Figure 3.

Our model predictions for $z_{\mathrm{RE}}(\mathbf{x})$ are constructed on density fields that are smoothed in the same way as the $z_{\mathrm{RE}}(\mathbf{x})$ fields, and the $b_{\mathrm{mz}}$ used are calculated directly from the corresponding simulation. The top panels of Figure 3 show that the $z_{\mathrm{RE}}(\mathbf{x})$ fields from the simulations and our model agree on large scales in their spatial distribution, structure, and evolution. Additionally, this illustrates that on smaller scales there are slight disagreements in the filament and void regions as well as the shape of the structures produced from the models, which are more filamentary than simulations. We find that our model for the ionization field at $z=8.1$, which approximately corresponds to $50 \%$ ionization in the $z_{\mathrm{RE}}(\mathbf{x})$ field, reproduces the simulations results at the same redshift (cf. Figure 3). The differences found between them are attributed to slightly different mean ionization fractions at $z=8.1$ and the steep changes in these fractions around this redshift. All the differences listed are attributed to the fact that the correlation is not exactly one at these scales, so using a model with a scale-dependent linear bias will not be perfect.

For a quantitative comparison of our model, we first compare simulation and model results for the cumulative distribution functions $(\mathrm{CDF})$ of $z_{\mathrm{RE}}(\mathbf{x})$ for an early and late scenario of reionization. Figure 4 shows that our model traces the simulation results well and only deviates from simulations at the very beginning and end of reionization, although this deviation is small. This corresponds to our model having inaccuracies in the

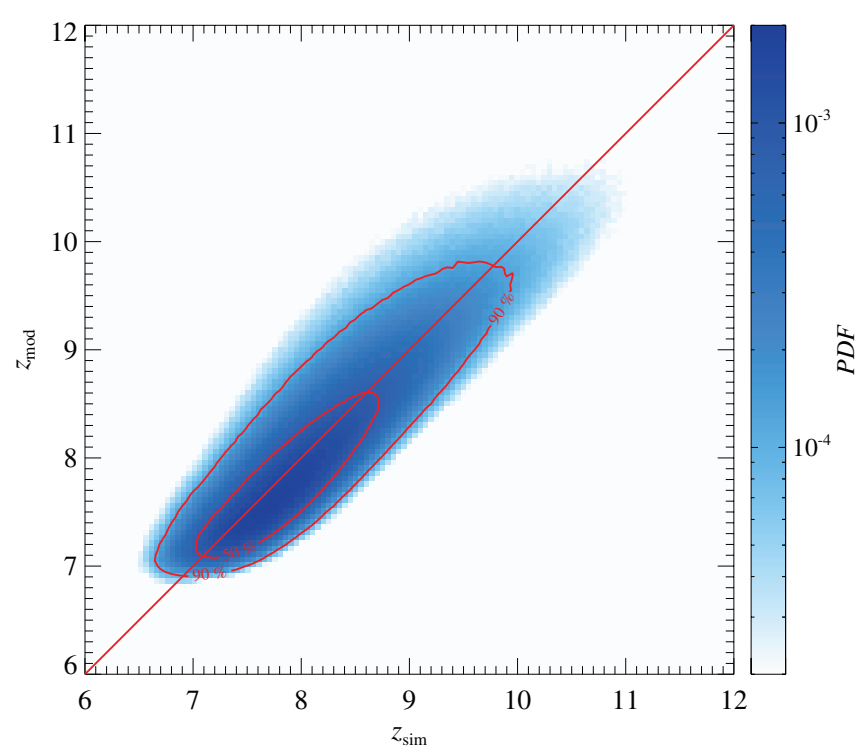

Figure 5. 2D PDF between the $z_{\mathrm{RE}}(\mathbf{x})$ fields from the RadHydro simulation for late reionization and our model predictions constructed from the same density field compared on the cell by cell level. The contours represent the cumulative probability distribution function for $50 \%$ and $90 \%$ of the cells in this comparison. Our model performs extremely well around $z=\bar{z}$ and slightly biases the $z_{\mathrm{RE}}(\mathbf{x})$ field to larger redshift values.

(A color version of this figure is available in the online journal.)

densest regions and in the voids, which is illustrated previously in Figure 3. The $z_{\mathrm{RE}}(\mathbf{x})$ values where the $\operatorname{CDF}(\bar{z})=0.5$ in our models show small offsets compared to the simulations $z_{\mathrm{RE}}(\mathbf{x})$ values for the same point in the CDF. We perform a cellby-cell comparison of the $z_{\mathrm{RE}}(\mathbf{x})$ fields between simulations and our model predictions, which are the most stringent tests. In Figure 5 we show the two-dimensional (2D) probability distribution function (PDF) (by the blue color scale) and CDF (red contours) for the late reionization scenario. A majority of the cells fall along the one-to-one line in this comparison with some scatter and they are clustered around $\bar{z}$ of this simulation. We find that $90 \%$ of the cells from the model are within $\sim 10 \%$ of the simulation value for their $z_{\mathrm{RE}}(\mathbf{x})$ values.

We compare the morphology of the ionization fields between the simulations and models statistically using the power spectra and cross correlations of these fields at a fixed redshift for the early reionization scenario. To calculate the ionization field at a given redshift, $z$, we set a cell at position $x$ to be ionized if $z_{\mathrm{RE}}(\mathbf{x}) \geqslant z$ (cf. Figure 3 bottom panels). In the left panel of Figure 6 we show the power spectra of ionization fields at $z=7.6, z=8.1$, and $z=8.6$, which correspond to global ionization fractions of $\sim 80 \%, 50 \%$, and $20 \%$, respectively. The simulation and model power spectra match in both the shape and amplitude at each redshift across all scales. We calculate the cross-correlation coefficient between the simulation and model ionization fields following Equation (6). The right panel of Figure 6 shows that the cross-correlation coefficient is close to 1 on large scales and drops to 0.8 at the smoothing scale. Despite that some differences are found between the images of the ionization field (cf. Figure 3 bottom panels), our model matches the simulations well in these statistical morphology tests. In fact, it is expected that the simulation and model have similar morphological statistics, since the model is constructed to reproduce these statistical properties.

After performing these stringent cell by cell and statistical tests, we did not find any large systematic biases in the mean 

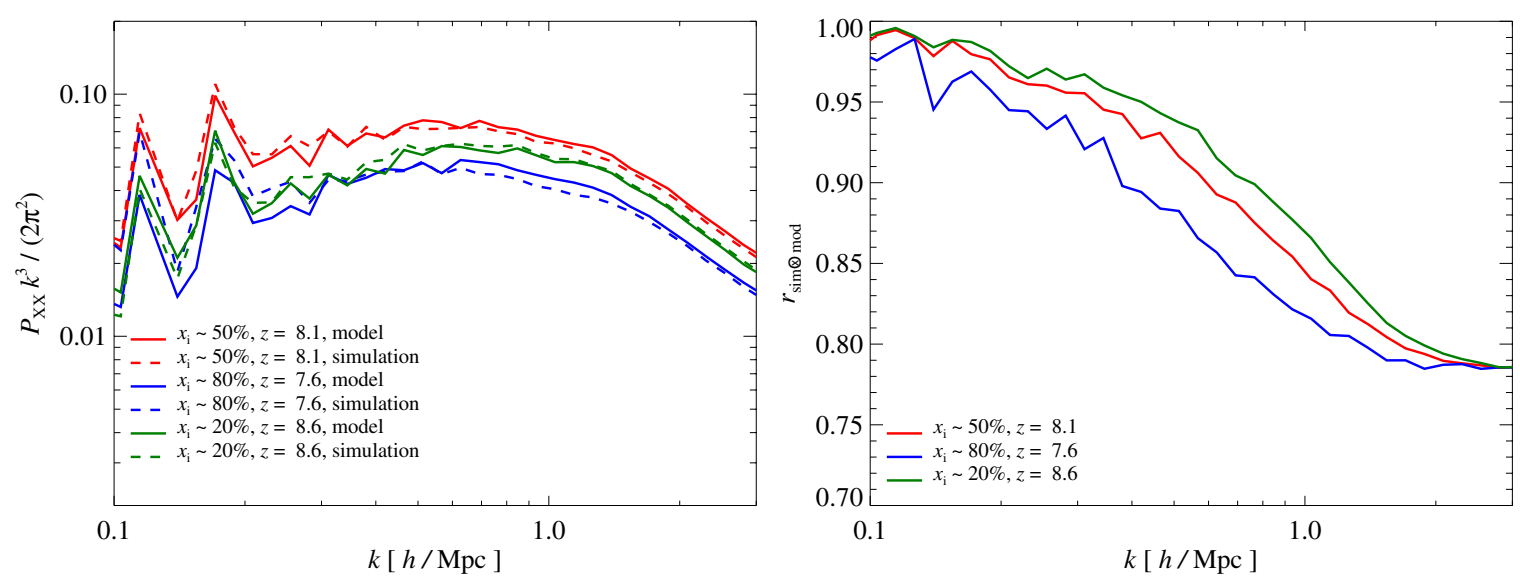

Figure 6. Left: the dimensionless power spectra of the ionization field from the simulation (dotted lines) and the model (solid lines) calculated at various redshifts. Right: the cross correlation, $r_{\text {sim } \otimes \text { mod }}$, of the ionization fields between the simulation and the model at various redshifts. Even though there are some differences seen in the ionization field (cf. Figure 3) statistically these fields are similar, which is illustrated by both power spectra and cross correlations for a range of redshifts. Under these comparison tests, our model performs as well as the previous semi-analytic models without having to adjust parameters at different redshifts.

(A color version of this figure is available in the online journal.)

values or the variations, but these small differences mentioned above are negligible compared to the uncertainties in the physics of reionization. We emphasize that no two radiative transfer hydrodynamic simulations nor semi-analytical models have ever been compared using all these stringent tests. Thus, our method of filtering the density field with a scale-dependent linear bias is a sufficient description of reionization especially on scales larger than $1 \mathrm{Mpc} h^{-1}$.

\section{RESULTS}

In this section, we present results on two physical aspects of the model, the ionization history and the correlation length between ionized regions, which is a proxy for bubble size. We explore the parameter space of Equation (8) for aspects, which provides physical understanding of the $z_{\mathrm{RE}}(\mathbf{x})$ field in relation to the parameters $k_{\mathrm{o}}, \alpha$, and $\bar{z}$.

\subsection{Ionization History}

We show the results for the mass weighted ionization history, $x_{\mathrm{i}}$, from our semi-analytic model for various parameter values of $k_{0}, \alpha$, and $\bar{z}$ in Figure 7. In this paper we present only the results for the mass weighted $x_{\mathrm{i}}$, since both the mass and volume weighted $x_{\mathrm{i}}$ have comparable redshift evolution with the slight difference being that the mass weighted $x_{\mathrm{i}}$ increases faster at higher redshifts than the volume weighted $x_{\mathrm{i}}$. The parameter $\bar{z}$ approximately sets the redshift where $x_{\mathrm{i}}=50 \%$. We find that for a fixed $k_{\mathrm{o}}$ and $\alpha$ the shape of $x_{\mathrm{i}}(z)$ about $\bar{z}$ is essentially independent of the $\bar{z}$ value and the percent differences between $\bar{z}=8,10,12$ are below $10 \%$ for $x_{\mathrm{i}} \gtrsim 10 \%$ (cf. Figure 7). The redshift evolution of the ionization history is set by $k_{\mathrm{o}}$ and $\alpha$, where increasing $\alpha$ shortens the duration of reionization and increasing $k_{\mathrm{o}}$ lengthens the duration of reionization (cf. Figure 8). When we decrease $\alpha$, the value of $b_{\mathrm{zm}}$ increases on small scales. Thus, we increase the variance in $\delta_{\mathrm{z}}$ and the duration of reionization, while decreasing $k_{\mathrm{o}}$ has opposite effect. These effects can be magnified by increasing one parameter while decreasing the other or vice versa. The physical interpretation of the parameters $\alpha$ and $k_{\mathrm{o}}$ can be understood by considering the following scenario in radiative transfer simulations or semi-analytic methods. Trac \& Cen (2007) showed that halos with $M \gtrsim 5 \times 10^{8} M_{\odot} h^{-1}$ contribute

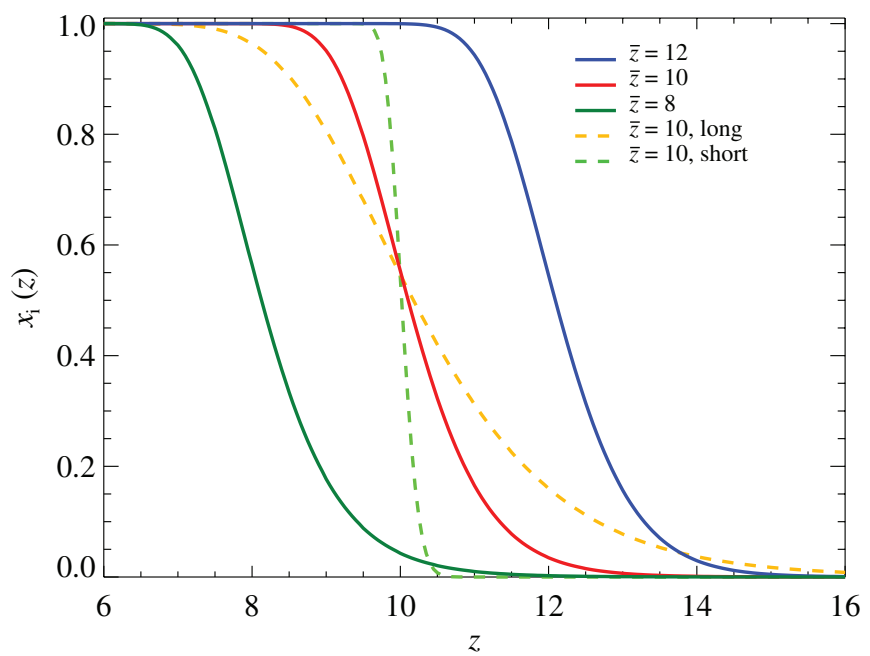

Figure 7. Ionization fraction as a function of redshift, $x_{\mathrm{i}}(z)$, for three fiducial parameter models with $\bar{z}=8,10$, and 12 (green, red, and blue lines, respectively) and the long and short duration reionization models (orange and light green dashed lines, respectively). The fiducial models have similar ionization histories, they are just shifted according to $\bar{z}$.

(A color version of this figure is available in the online journal.)

approximately $50 \%$ to the total ionizing photon budget. We can push the characteristic halo mass scale to higher values by increasing the minimum mass of halos hosting radiation sources or by increasing the star formation efficiency or radiation escape fraction preferentially in higher-mass halos. As a result, small-scale structure decreases, the characteristic size of ionized regions increases, and overall the duration of reionization becomes shorter (e.g., McQuinn et al. 2007). These changes are effectively equivalent to increasing $\alpha$ or decreasing $k_{0}$. We will further explore the connection between the parameters of the bias model and the parameters of source and sink models in radiative transfer simulations or semi-analytic methods in future work.

Similar to previous work (e.g., Zahn et al. 2012), we define two measures for the duration of reionization, $\Delta_{\mathrm{z}} \equiv z\left(x_{\mathrm{i}}=\right.$ $25 \%)-z\left(x_{\mathrm{i}}=75 \%\right)$ and $\Delta_{\mathrm{z}} \equiv z\left(x_{\mathrm{i}}=5 \%\right)-z\left(x_{\mathrm{i}}=95 \%\right)$. In general, semi-analytic models and simulations exclude the 

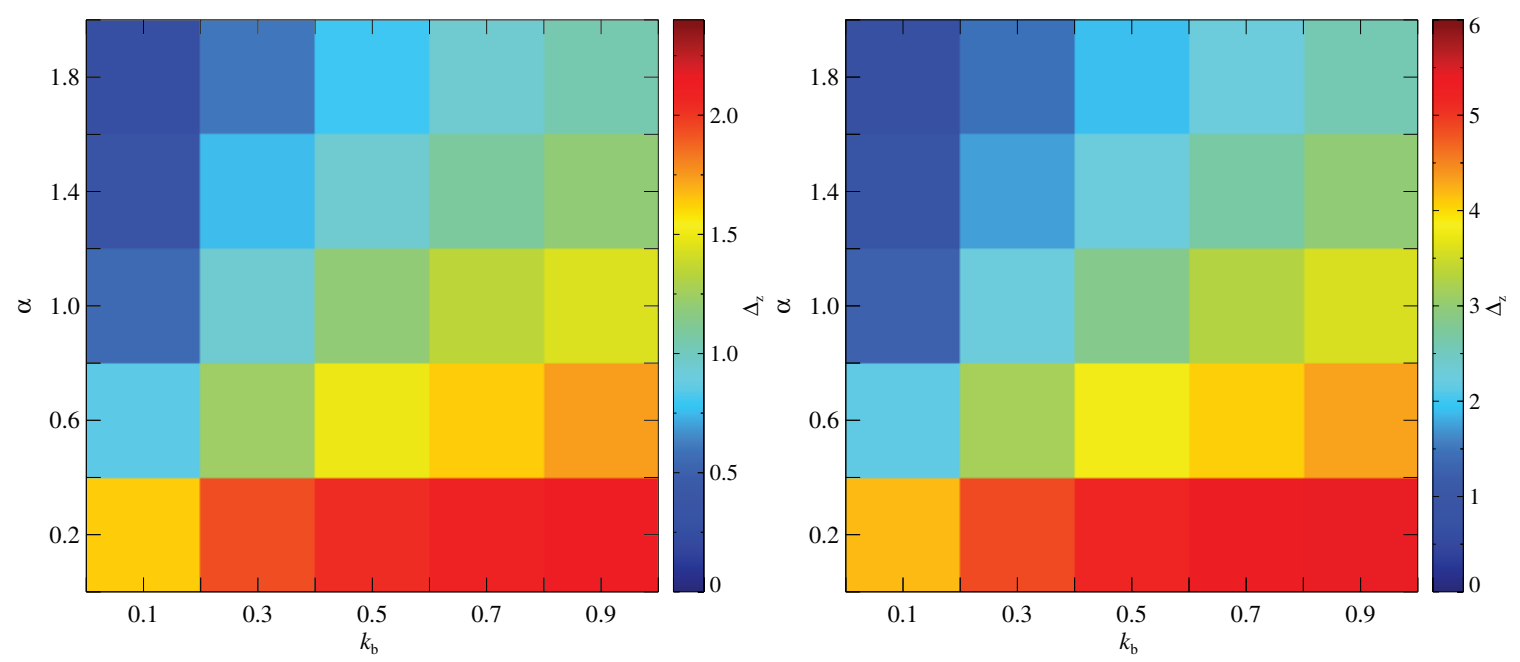

Figure 8. Parameter grids of $k_{\mathrm{o}}$ and $\alpha$ for the width of reionization, $\Delta_{\mathrm{z}}$. In the left panel $\Delta_{\mathrm{z}} \equiv z\left(x_{\mathrm{i}}=25 \%\right)-z\left(x_{\mathrm{i}}=75 \%\right)$ and in the right panel $\Delta_{\mathrm{z}} \equiv z\left(x_{\mathrm{i}}=5 \%\right)-z\left(x_{\mathrm{i}}=95 \%\right)$. Increasing $k_{\mathrm{o}}$ extends the duration of reionization, while increasing $\alpha$ shortens the duration of reionization. The trends for $\Delta_{\mathrm{z}}\left(k_{\mathrm{o}}, \alpha\right)$ are independent of the definition of $\Delta_{\mathrm{z}}$. There are degenerate combinations of $k_{\mathrm{o}}$ and $\alpha$ that give the same values for $\Delta_{\mathrm{z}}$.

(A color version of this figure is available in the online journal.)
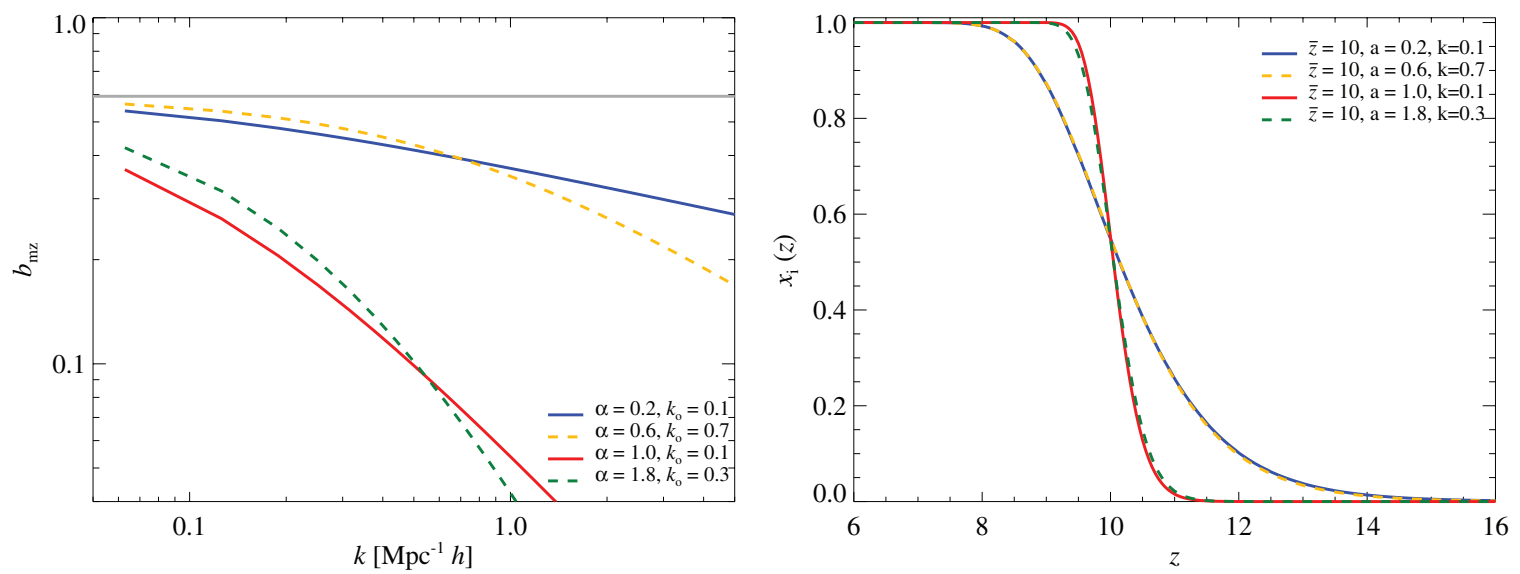

Figure 9. Left: the $b_{\mathrm{mz}}$ functions for two examples of degenerate pair combinations of $k_{\mathrm{o}}$ and $\alpha$ with similar $\Delta_{\mathrm{z}}$ at fixed $\bar{z}=10$ (cf. Figure 8). Right: the $x_{\mathrm{i}}(z)$ corresponding to the same pair combinations. Even though the $b_{\mathrm{mz}}$ functions of these degenerate pair combinations are different, they have similar $x_{\mathrm{i}}(z)$.

(A color version of this figure is available in the online journal.)

early and late times of reionization when defining $\Delta_{\mathrm{z}}$, since it is difficult to capture the small-scale physical processes at these times. Figure 8 shows how $k_{\mathrm{o}}$ and $\alpha$ affect $\Delta_{\mathrm{z}}$. The trends in $x_{\mathrm{i}}$ from varying the values of $k_{\mathrm{o}}$ and $\alpha$ are the same for $\Delta_{\mathrm{z}}$ and independent of the definition for $\Delta_{\mathrm{z}}$. Currently there are upper limits for $\Delta_{\mathrm{z}}$ from CMB small-scale measurements (Zahn et al. 2012; Mesinger et al. 2012), independent of definition and lower limits for $\Delta_{\mathrm{Z}}$ from global $21 \mathrm{~cm}$ observations (Bowman \& Rogers 2012). With a few exceptions, all our models fall well within the constrained region of parameter space for $\Delta_{\mathrm{z}}$ from these observations, although we emphasize that the conversion from observation to $\Delta_{\mathrm{z}}$ is model dependent.

There are degeneracies in both $x_{\mathrm{i}}(z)$ and $\Delta_{\mathrm{z}}$ in our parametric bias model. Figure 9 shows two examples of parameters pairs values of $\alpha=0.2, k_{\mathrm{o}}=0.1$ and $\alpha=0.6, k_{\mathrm{o}}=0.7$ that have different bias functions, but they have similar ionization histories (cf. Figure 9) and $\Delta_{z}$ values (cf. Figure 8). These degeneracies are a problem if one wants to relate any $x_{\mathrm{i}}(z)$ observable to the underlying parameters of our model. It is possible to use higher order statistics than $\Delta_{\mathrm{z}}$, i.e., beyond the variance, to differentiate between these degenerate models, but this does not add physical understanding of how $\alpha$ and $k_{\mathrm{o}}$ affect the EoR. Any measures or proxies for the typical sizes of ionizing regions will differentiate between the degenerate pairs of $\alpha$ and $k_{\mathrm{o}}$, while providing a more physical understanding of the impact these parameters have on the EoR.

\subsection{Correlation Length between Ionized Regions}

For each of the EoR models, we follow the same procedure for constructing the ionization field and measuring the $3 \mathrm{D}$ power spectrum used to make Figure 6. Since the power spectrum is expected to peak on scales where the ionized regions are the most correlated, we compress all the information from these spectra into the typical correlation length between ionized regions, $\lambda_{\mathrm{i}}$. We find the wavenumber, $k_{\mathrm{i}}$, where the spectrum peaks and we define the typical correlation length between ionized regions to be $\lambda_{\mathrm{i}}=2 \pi / k_{\mathrm{i}}$.

The value of $\lambda_{\mathrm{i}}$ is a proxy for the typical ionized bubble size. We find that the largest $\lambda_{\mathrm{i}}$ values appear around $\bar{z}$. After $\bar{z}, \lambda_{\mathrm{i}}$ no longer measures the typical correlation length between ionized regions; instead it measures the typical correlation between neutral regions (i.e., typical size of neutral clouds). The redshift evolution of $\lambda_{\mathrm{i}}$ for fixed $k_{\mathrm{o}}$ and $\alpha$ is independent of $\bar{z}$ (cf. Figure 10). At a fixed $\bar{z}=10$ the value for $\lambda_{\mathrm{i}}$ ranges from 


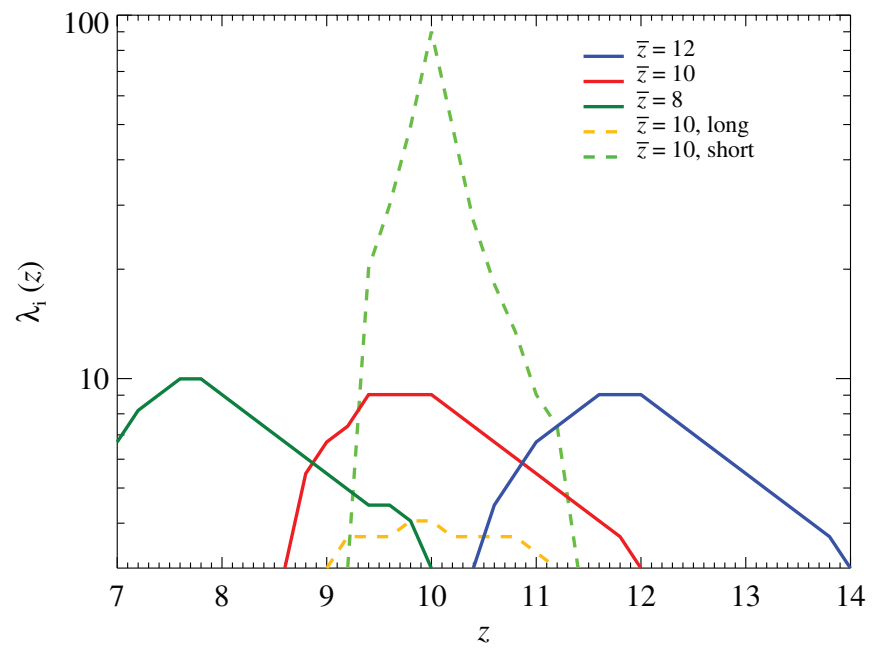

Figure 10. Typical correlation length between ionized regions, $\lambda_{i}$, for three fiducial parameter models with $\bar{z}=8,10$, and 12 (green, red, and blue lines, respectively) and the long and short duration reionization models (orange and light green dashed line, respectively). The values of $\lambda_{\mathrm{i}}(z)$ for the fiducial models is independent of $\bar{z}$. Comparing the values at $z=10$, where all models with $\bar{z}=10$ have similar $x_{\mathrm{i}}$ values, there is a significant difference between $\lambda_{\mathrm{i}}(z)$ for the long and short duration reionization.

(A color version of this figure is available in the online journal.)

approximately 3-90 Mpc $h^{-1}$ between our long and short duration reionization models (cf. Figure 10). Complementary to the results shown in Section 4.1, we find that the long duration reionization model has small correlation lengths between ionized regions, while the short duration reionization model has large correlation lengths between ionized regions. We chose to compare our models at $\bar{z}$ when studying the parameter space of $k_{\mathrm{o}}$ and $\alpha$, since at $\bar{z}$ all these models have approximately the same ionization fraction $(\sim 50 \%)$. In Figure 11 we show the parameter space grid for $\lambda_{\mathrm{i}}$ a function of $k_{\mathrm{o}}$ and $\alpha$ for a fixed $\bar{z}=10$. The same trends for $\Delta_{\mathrm{z}}$ as a function of $k_{\mathrm{o}}$ and $\alpha$ are found in $\lambda_{\mathrm{i}}$. However, the degenerate combinations of $k_{\mathrm{o}}$ and $\alpha$ that give similar $\Delta_{\mathrm{z}}$ values have different $\lambda_{\mathrm{i}}$ values (cf. Figure 8 and 11). Thus, some of the model degeneracies in observations that only measure $\Delta_{\mathrm{z}}$ will begin to be broken by any observation that measures $\lambda_{\mathrm{i}}$ such as the $21 \mathrm{~cm}$ signal or $\mathrm{kSZ}$ power spectrum.

\section{DISCUSSION}

Semi-analytic models are clearly important tools for understanding the EoR. They are able to quickly explore the large parameter space of unknown and unconstrained physics in an attempt to quantify how these unknown physical processes impact observables from the EoR. There is an abundance of semianalytic models (e.g., Furlanetto et al. 2004; Zahn et al. 2007; Alvarez et al. 2009; Choudhury et al. 2009) that compute an ionization field from a density field (initial conditions or $N$-body), which rely on a couple of free parameters such as an efficiency $\zeta$ to model the unknown and unresolved physics of the EoR. When comparing against simulations (e.g., Zahn et al. 2011), these free parameters are tuned such that the models match the ionization fractions of the radiative transfer simulations for a specific redshift. If this redshift is changed, the parameters change. Since the parameter values in the models do not capture the same redshift evolution as the simulations, it is difficult to directly compare them to the sub-grid model values used in simulations and their physical interpretations are diminished. Our

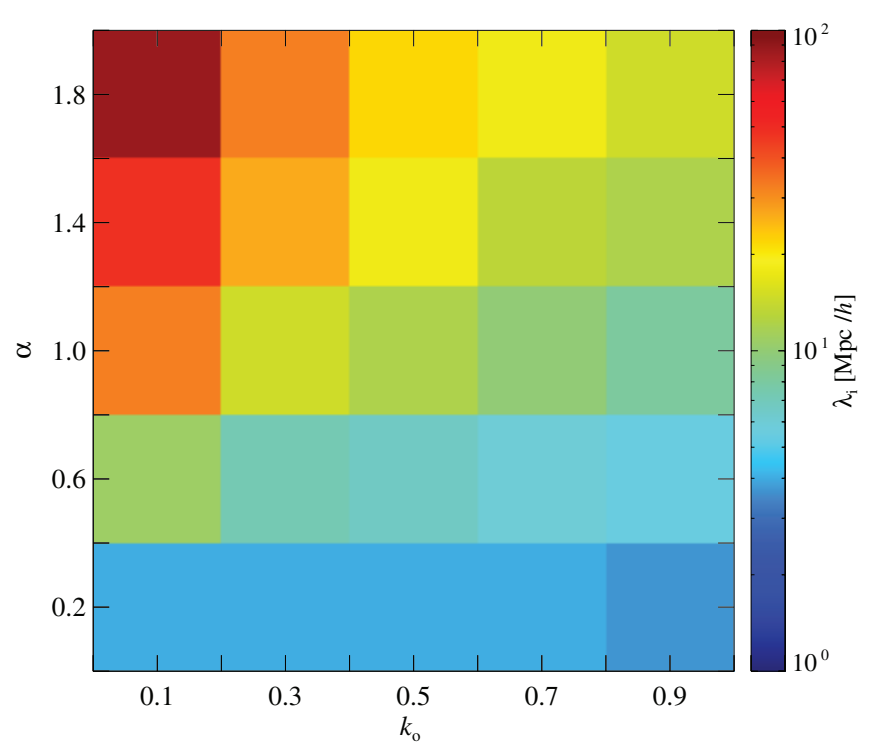

Figure 11. Parameter grid of $k_{\mathrm{o}}$ and $\alpha$ for the typical correlation length between ionized regions, $\lambda_{\mathrm{i}}$ for $\bar{z}=10$ and at $z=\bar{z}$. The previous degenerate combinations of $k_{\mathrm{o}}$ and $\alpha$ that gave the same values for $\Delta_{\mathrm{z}}$ give different values for $\lambda_{\mathrm{i}}$; for example, the $\lambda_{\mathrm{i}}$ values for the parameters values $\alpha=0.2, k_{\mathrm{o}}=0.1$ and $\alpha=0.6, k_{\mathrm{o}}=0.7$ are different.

(A color version of this figure is available in the online journal.)

model differs in that all the complex non-linear physics within the simulations are encapsulated in the parametric form $b_{\mathrm{mz}}$. The direct comparison between model and simulation is trivial, since $b_{\mathrm{mz}}$ is computed from the simulations and inserted into our model. In principle, when we calibrated $b_{\mathrm{mz}}$ to simulations there is no loss of accuracy on large scales $\left(>1 \mathrm{Mpc} h^{-1}\right)$ and there is only one free parameter, $\bar{z}$. Additionally, our model is extremely quick and easily applicable to large $N$-body simulations, since it requires only two fast Fourier transforms to calculate an ionization field, with the rate limiting step being the input and output of the large density and ionization fields, respectively.

The model we proposed is based on an inside-out scenario for reionization, like several other semi-analytic models, which assumes that the first generation of galaxies are the dominant sources for the ionizing photons. This model does not capture exotic reionization scenarios, like those where void regions reionize first. Furthermore, the simulations that our model is derived from do not include ionizing photons from Population III stars or first X-ray sources like high redshift quasars (e.g., Wyithe \& Cen 2007; Trac \& Cen 2007). The impact of these alternate sources for ionizing photons on the EoR is still an open question (e.g., Ahn et al. 2012; Visbal \& Loeb 2012; Feng et al. 2013). In future work, we will look into implementing higher order $b_{\mathrm{mz}}$ and $r_{\mathrm{mz}}$ statistics, which should increase the ability of our model to accurately map simulations onto larger and smaller scales; and including ways to incorporate exotic reionization scenarios, thus increasing the versatility of the model.

\section{CONCLUSIONS}

We present a novel semi-analytic model for calculating the redshift evolution of the ionization field during the EoR that is fast and easily applicable to large volume $N$-body simulations. Our model is motivated by and calibrated to RadHydro simulations, which show there is a strong correlation between the density and the reionization redshift fields on scales $\gtrsim 1 \mathrm{Mpc} h^{-1}$. A simple filter (cf. Equation (10)) is convolved 
with a non-linear density field at $z=\bar{z}$ to obtain a $z_{\mathrm{RE}}(\mathbf{x})$, which depends mainly on the parameters $k_{\mathrm{o}}, \alpha$, and $\bar{z}$. The number of parameters is reduced to one, $\bar{z}$ (essentially sets the mean reionization redshift), when the values of $k_{\mathrm{o}}$ and $\alpha$, are fit to simulation results.

We found that this model performed well on large scales when we compare it directly to RadHydro simulations. Three of the comparisons we performed were stringent tests of the simulated and modeled $z_{\mathrm{RE}}(\mathbf{x})$ fields. (1) We compared slices of the $z_{\mathrm{RE}}(\mathbf{x})$ field between the simulation and model illustrating the minor differences in the evolution of the $z_{\mathrm{RE}}(\mathbf{x})$ field. (2) We compared the cumulative distribution of the $z_{\mathrm{RE}}(\mathbf{x})$ values, which again yielded minor differences between the simulation and model. (3) We constructed a 2D probability distribution function for a cellby-cell comparison of $z_{\mathrm{RE}}(\mathbf{x})$ between the simulation and model that showed that $90 \%$ of all $z_{\mathrm{RE}}(\mathbf{x})$ values in the model were within $10 \%$ of the simulation values. Given that all the slight differences between the simulation and model were well below the uncertainty in the details of the astrophysical processes at work during the EoR, the model we proposed is a great tool for incorporating radiation hydrodynamic physics of reionization into large $N$-body simulations.

We introduce a physical understanding of the parameters $k_{\mathrm{O}}$ and $\alpha$ by comparing the ionization histories, $x_{\mathrm{i}}$, and typical correlation length between ionized regions, $\lambda_{\mathrm{i}}$, for various combinations of $k_{\mathrm{o}}$ and $\alpha$. Decreasing the parameter $k_{\mathrm{o}}$ shortens the duration of the EoR, while increasing $k_{\mathrm{o}}$ lengthens the duration of the EoR, which is a direct result of changing the variance of $\delta_{\mathrm{z}}$. The parameter $\alpha$ has the opposite behavior, i.e., increasing $\alpha$ shortens the duration of the EoR and vice versa. For the values of $\bar{z}$ we explored, these physical interpretations of $k_{\mathrm{o}}$ and $\alpha$ are independent of the $\bar{z}$ value. There are degenerate combinations of $k_{\mathrm{o}}$ and $\alpha$ that produce nearly identical $x_{\mathrm{i}}(z)$. These degeneracies are broken by $\lambda_{\mathrm{i}}$ and allow one to further differentiate between parameter combinations. Thus, degeneracy in observables, which depend on $x_{\mathrm{i}}(z)$, can be broken when combined with observables that depend on the $\lambda_{\mathrm{i}}$. Similar to $x_{\mathrm{i}}(z)$, the values for $\lambda_{\mathrm{i}}(z)$ at fixed $k_{\mathrm{o}}$ and $\alpha$ are practically independent of $\bar{z}$. It is necessary to compare values for $\lambda_{\mathrm{i}}(z)$ for varying $k_{\mathrm{o}}$ and $\alpha$ at fixed $x_{\mathrm{i}}$, so comparing at $z=\bar{z}$ is a natural choice. For a fixed $z=\bar{z}$, we demonstrate that smaller $\lambda_{\mathrm{i}}$ values are obtained by increasing $k_{\mathrm{o}}$ or decreasing $\alpha$ while larger $\lambda_{\mathrm{i}}$ are obtained by decreasing $k_{\mathrm{o}}$ or increasing $\alpha$, with values for $\lambda_{\mathrm{i}}$ ranging from $\sim 3$ to $90 \mathrm{Mpc} h^{-1}$. In summary, any combination of $k_{\mathrm{o}}$ and $\alpha$ that extends the function $b_{\mathrm{mz}}$ to large values of $k$ will increase the amount of small scale structure, thus increasing $\Delta_{\mathrm{z}}$ and decreasing $\lambda_{\mathrm{i}}$.

Our method is an accurate and fast tool for exploring galactic reionization on large scales and going forward we will use it to make testable predictions for CMB and $21 \mathrm{~cm}$ observables.

N.B. is supported by a McWilliams Center for Cosmology Postdoctoral Fellowship made possible by Bruce and Astrid McWilliams. We thank Aravind Natarajan, Paul La Plante, Jonathan Sievers, and Christian Reichardt for useful discussions. We thank N. Gnedin for his compilation of the ionization and recombination rates and D. Schaerer for the Population II spectral energy distributions. H.T. is supported in part by NSF grant AST-1109730. R.C. is supported in part by NSF grant AST-1108700 and NASA grant NNX12AF91G. A.L. is supported in part by NSF grant AST-0907890 and NASA grants NNX08AL43G and NNA09DB30A. The simulations were performed at the Pittsburgh Supercomputing Center (PSC) and the Princeton Institute for Computational Science and
Engineering (PICSciE). We thank Roberto Gomez and Rick Costa at the PSC and Bill Wichser at PICSciE for invaluable help with computing.

\section{REFERENCES}

Ahn, K., Iliev, I. T., Shapiro, P. R., et al. 2012, ApJL, 756, L16

Altay, G., Croft, R. A. C., \& Pelupessy, I. 2008, MNRAS, 386, 1931

Alvarez, M. A., Busha, M., Abel, T., \& Wechsler, R. H. 2009, ApJL, 703, L167

Alvarez, M. A., Komatsu, E., Doré, O., \& Shapiro, P. R. 2006, ApJ, 647, 840

Aubert, D., \& Teyssier, R. 2008, MNRAS, 387, 295

Barkana, R., \& Loeb, A. 2004, ApJ, 609, 474

Battaglia, N., Natarajan, A., Trac, H., Cen, R., \& Loeb, A. 2013, ApJ, in press Bond, J. R., Cole, S., Efstathiou, G., \& Kaiser, N. 1991, ApJ, 379, 440

Bowman, J. D., Morales, M. F., \& Hewitt, J. N. 2005, BAAS, 37, 1217 Bowman, J. D., \& Rogers, A. E. E. 2012, arXiv:1209.1117

Choudhury, T. R., Haehnelt, M. G., \& Regan, J. 2009, MNRAS, 394, 960 Ciardi, B., Ferrara, A., Marri, S., \& Raimondo, G. 2001, MNRAS, 324, 381 Croft, R. A. C., \& Altay, G. 2008, MNRAS, 388, 1501

Fan, X., Strauss, M. A., Becker, R. H., et al. 2006, AJ, 132, 117

Feng, Y., Croft, R. A. C., Di Matteo, T., \& Khandai, N. 2013, MNRAS, 429, 1554

Finlator, K., Özel, F., \& Davé, R. 2009, MNRAS, 393, 1090

Furlanetto, S. R., Oh, S. P., \& Briggs, F. H. 2006, PhR, 433, 181

Furlanetto, S. R., Zaldarriaga, M., \& Hernquist, L. 2004, ApJ, 613, 1

Geil, P. M., \& Wyithe, J. S. B. 2008, MNRAS, 386, 1683

Gnedin, N. Y., \& Abel, T. 2001, NewA, 6, 437

Gnedin, N. Y., \& Fan, X. 2006, ApJ, 648, 1

Gruzinov, A., \& Hu, W. 1998, ApJ, 508, 435

Harker, G., Zaroubi, S., Bernardi, G., et al. 2010, MNRAS, 405, 2492

Iliev, I. T., Mellema, G., Pen, U.-L., et al. 2006, MNRAS, 369, 1625

Iliev, I. T., Pen, U.-L., Bond, J. R., Mellema, G., \& Shapiro, P. R. 2007, ApJ, 660,933

Knox, L., Scoccimarro, R., \& Dodelson, S. 1998, PhRvL, 81, 2004

Larson, D., Dunkley, J., Hinshaw, G., et al. 2011, ApJS, 192, 16

Lidz, A., Oh, S. P., \& Furlanetto, S. R. 2006, ApJL, 639, L47

Loeb, A., \& Furlanetto, S. 2013, The First Galaxies in the Universe (Princeton: Princeton Univ. Press)

Maselli, A., Ferrara, A., \& Ciardi, B. 2003, MNRAS, 345, 379

McQuinn, M., Furlanetto, S. R., Hernquist, L., Zahn, O., \& Zaldarriaga, M. 2005, ApJ, 630, 643

McQuinn, M., Lidz, A., Zahn, O., et al. 2007, MNRAS, 377, 1043

Mellema, G., Iliev, I. T., Pen, U.-L., \& Shapiro, P. R. 2006, MNRAS, 372, 679

Mellema, G., Koopmans, L. V. E., Abdalla, F. A., et al. 2013, ExA, 36, 235

Mesinger, A., \& Furlanetto, S. 2007, ApJ, 669, 663

Mesinger, A., Furlanetto, S., \& Cen, R. 2011, MNRAS, 411, 955

Mesinger, A., McQuinn, M., \& Spergel, D. N. 2012, MNRAS, 422, 1403

Morales, M. F., \& Wyithe, J. S. B. 2010, ARA\&A, 48, 127

Natarajan, A., Battaglia, N., Trac, H., Li Pen, U., \& Loeb, A. 2013, ApJ, in press Oh, S. P., \& Furlanetto, S. R. 2005, ApJL, 620, L9

Parsons, A. R., Backer, D. C., Foster, G. S., et al. 2010, AJ, 139, 1468

Pen, U.-L., Chang, T.-C., Hirata, C. M., et al. 2009, MNRAS, 399, 181

Petkova, M., \& Springel, V. 2009, MNRAS, 396, 1383

Santos, M. G., Cooray, A., Haiman, Z., Knox, L., \& Ma, C.-P. 2003, ApJ, 598,756

Santos, M. G., Ferramacho, L., Silva, M. B., Amblard, A., \& Cooray, A. 2010, MNRAS, 406, 2421

Schaerer, D. 2003, A\&A, 397, 527

Scoccimarro, R., \& Sheth, R. K. 2002, MNRAS, 329, 629

Scott, D., \& Rees, M. J. 1990, MNRAS, 247, 510

Shapiro, P. R., Iliev, I. T., \& Raga, A. C. 2004, MNRAS, 348, 753

Shaver, P. A., Windhorst, R. A., Madau, P., \& de Bruyn, A. G. 1999, A\&A, 345,380

Thomas, R. M., Zaroubi, S., Ciardi, B., et al. 2009, MNRAS, 393, 32

Trac, H., \& Cen, R. 2007, ApJ, 671, 1

Trac, H., Cen, R., \& Loeb, A. 2008, ApJL, 689, L81

Trac, H., \& Pen, U.-L. 2004, NewA, 9, 443

Trac, H. Y., \& Gnedin, N. Y. 2011, ASL, 4, 228

Valageas, P., Balbi, A., \& Silk, J. 2001, A\&A, 367, 1

Visbal, E., \& Loeb, A. 2012, JCAP, 05, 007

Wyithe, J. S. B., \& Cen, R. 2007, ApJ, 659, 890

Zahn, O., Lidz, A., McQuinn, M., et al. 2007, ApJ, 654, 12

Zahn, O., Mesinger, A., McQuinn, M., et al. 2011, MNRAS, 414, 727

Zahn, O., Reichardt, C. L., Shaw, L., et al. 2012, ApJ, 756, 65

Zahn, O., Zaldarriaga, M., Hernquist, L., \& McQuinn, M. 2005, ApJ, 630, 657

Zaldarriaga, M., Furlanetto, S. R., \& Hernquist, L. 2004, ApJ, 608, 622 